\title{
Spatial Distribution of the Geomagnetic Spectral Composition for Disturbed Days
}

\author{
Wallace H. CAMPBeLL \\ U.S. Geological Survey, Federal Center, Denver, Colorado, U.S.A.
}

(Received June 7, 1976; Revised September 20, 1976)

\begin{abstract}
Seven geomagnetically disturbed days were studied at a number of stations in the American Hemisphere for the purpose of determining the systematic spectral composition changes with activity level, $A p$, and geomagnetic latitude. The spectral amplitudes, $A$, at 5, 10, 30, 60, and 120-min period, $T$, from a Fourier analysis of magnetograms showed a linear relationship with $A p$ that varied with $T$. There was an auroral latitude maximum of activity at all periods (which shifted poleward for lower $A p$ ), a low latitude minimum, and a daytime equatorial enhancement of the spectra. Calling $m$ the spectral slope from $A=k T^{m}$, where $k$ is a constant, the slopes were determined for the period ranges of 5 to 10,10 to 60 , and 60 to $240 \mathrm{~min}$. Average slope values in these ranges were about $2.4,1.0$, and 0.8 respectively. The 10 to 60-min slope values increased linearly with increasing $A P$ and decreased at auroral latitudes.
\end{abstract}

\section{Introduction}

This is a spectral composition study of geomagnetically disturbed days for the purpose of establishing the predictable behavior of spatial and temporal characteristics of the geomagnetic field variation spectra so that such information may be available for other investigations of special geophysical interest. This study complements an earlier publication (CAMPBELL, 1976) which focused upon the monthly average spectral characteristics.

Seven disturbed day events (Table 1) were selected for this study because the dates of the events included a wide range of activity levels; a large number of geomagnetic observatories about the North American continent had recorded the activity and magnetic field records had already been digitized and were available as computer tapes from the World Data Center A. Table 2 is a list of observatory locations in geomagnetic latitude order, a major parameter for this study. A map showing the location of these stations was given in the earlier paper (op. cit.). The digitized records were 2.5 -min interval scalings of the three orthogonal, $D, H$, and $Z$, geomagnetic field components. 
Table 1. Geomagnetically active events.

\begin{tabular}{cccc}
\hline Date & $\begin{array}{c}A p \\
\text { index }^{11}\end{array}$ & $\begin{array}{c}N \\
(\text { number/year })^{2)}\end{array}$ & $\begin{array}{c}\text { UT } \\
(60-\text { min max })^{3)}\end{array}$ \\
\hline 8 March 1970 & 149 & $1 / 2$ & 1600 \\
16 June 1965 & 73 & 3 & 1400 \\
18 April 1965 & 68 & 3 & 0600 \\
7 February 1965 & 31 & 18 & 0400 \\
4 March 1965 & 21 & 44 & 0600 \\
18 August 1965 & 21 & 44 & 1800 \\
6 November 1965 & 17 & 71 & 1000 \\
\hline
\end{tabular}

1) Daily average geomagnetic activity index.

2) Expected average number of days per year with greater than given $A p$.

3) Hour of maximum amplitude for 60 -min spectral component.

Table 2. Geomagnetic station list.

\begin{tabular}{|c|c|c|c|c|c|c|}
\hline \multirow{2}{*}{ Code } & \multirow{2}{*}{ Station } & \multicolumn{2}{|c|}{ Geomagnetic } & \multirow{2}{*}{$\frac{\text { Invariant }}{\text { Latitude }}$} & \multicolumn{2}{|c|}{ Geographic } \\
\hline & & Latitude & Longitude & & Latitude & Longitude \\
\hline $\mathrm{HU}$ & Huancayo & -0.64 & 354.27 & 12.68 & -12.05 & 284.67 \\
\hline $\mathrm{TT}^{11}$ & Tatuoca & 9.51 & 21.25 & 16.16 & -1.20 & 311.48 \\
\hline $\mathrm{FQ}^{21}$ & Fuquene & 16.91 & 355.57 & 21.44 & 5.47 & 286.27 \\
\hline SJ & San Juan & 29.57 & 3.63 & 32.43 & 18.12 & 293.85 \\
\hline $\mathrm{TU}^{2)}$ & Tucson & 40.48 & 312.72 & 39.38 & 32.25 & 249.17 \\
\hline DS & Dallas & 42.98 & 328.29 & 42.98 & 32.98 & 263.25 \\
\hline FR & Fredericksburg & 49.54 & $350 . \overline{42}$ & 50.72 & 38.20 & 282.63 \\
\hline VI & Victoria & 54.27 & 293.51 & 53.76 & 48.52 & 236.58 \\
\hline SI & Sitka & 60.09 & 275.86 & 59.60 & 57.07 & 224.67 \\
\hline ME & Meanook & 61.89 & 301.62 & 62.26 & 54.62 & 246.67 \\
\hline $\mathrm{CO}$ & College & 64.73 & 256.99 & 64.63 & 64.87 & 212.17 \\
\hline FC & Ft. Churchill & 68.69 & 323.46 & 69.80 & 58.80 & 265.90 \\
\hline BW & Barrow & 68.95 & 241.55 & 69.43 & 71.30 & 203.25 \\
\hline $\mathrm{BL}$ & Baker Lake & 73.81 & 315.98 & 74.79 & 64.33 & 263.97 \\
\hline $\mathrm{GO}^{11}$ & Godhavn & 79.78 & 33.33 & 76.89 & 69.23 & 306.48 \\
\hline $\mathrm{AT}$ & Alert & 85.87 & 167.29 & 86.74 & 82.50 & 297.50 \\
\hline $\mathbf{T H}^{2)}$ & Thule & 88.95 & 3.56 & 86.24 & 77.48 & 290.83 \\
\hline
\end{tabular}

Four-hour data samples were analyzed using the Fast Fourier Transform (FFT) technique of CoOLEY et al. (1969), to provide spectral displays for periods $(T)$ of the field variations between $5 \mathrm{~min}$ and $4 \mathrm{hr}$. The limiting Nyquist period of data samples was $T_{\mathrm{N}}=5 \mathrm{~min}$; reliable spectral amplitude values were obtained at least above $T=T_{\mathrm{N}} 5 / 4=6.25 \mathrm{~min}$ (OTNES and ENOCHSON, 1972). Linear trends in the data were removed before the FFT analysis by substracting a straight line between the average of five points at the beginning and end of the sample. 
These trends are primarily the long-term secular change of the Earth's main field as well as seasonal and day-to-day solar-terrestrial activity variations in the magnetosphere. End values of the data sample were tapered with a standard "cosine bell" curve. For the very rare occasions when some points were missing in the magnetogram scalings, estimated values were filled in linearly between the last and next real data point for those cases when the gap was not more than one hour; for longer gaps the station's data were omitted from the analysis.

For all the stations indicated in Table 2 spectra were determined every $2 \mathrm{hr}$ beginning at 2200 UT preceding the selected day, for 13 successive intervals, covering the period to 0200 UT following the selected day. Each frame of the spectral display series was identified by the field component, date, station, and central hour of each 4-hr interval as illustrated in Fig. 1 for quiet and active examples. Such displays were plotted in log-log coordinates because the geomagnetic field amplitudes, $A$, for periods, $T$, in this study range may be approximately represented as $A=k T^{m}$ where $k$ and $m$ are constants. For such an equation $\log A=k^{\prime}+m \log T$ is a linear display in which $m$ is called the spectral slope and the intercept constant $k^{\prime}=\log k$.

Fluctuating noise analysis procedures (ScHWARTZ, 1959) were assumed for the processing of these $D, H$, and $Z$ magnetic field records. In this technique, the spectral amplitudes one obtains are the Fourier component amplitude terms. These values, in units of gamma (gamma $=10^{-9}$ Tesla), are the amplitudes given throughout this paper. Such a presentation allows the reader to visualize the relative sizes of the geomagnetic field changes at different stations for various parts of the spectra. However, the numerical values are dependent upon the

\section{4-HR SPECTRA H COMPONENT}

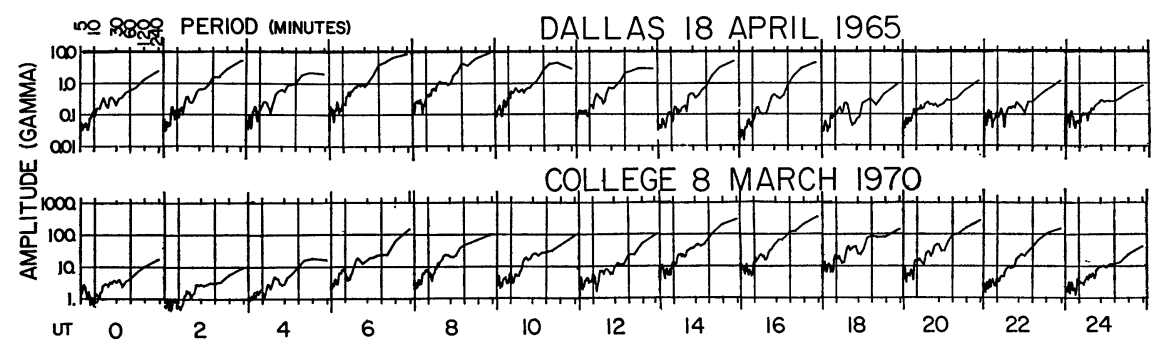

Fig. 1. Series of 4-hr spectra of the geomagnetic $H$-component field variations at Dallas on 18 April 1965 and College on 8 March 1970. The time shown identified the central UT hours of each analysis interval. The spectral period (min) and corresponding Fast Fourier Transform amplitudes (gamma) are plotted on logarithmic scales. The specially marked periods indicate the selected values used for contour and projection plotting of the composite station data. Vertical lines within each hour group divide 3 period ranges within which the spectral slope, $m$, was scaled. 
data sample duration and number of scaled values within the sample. The Fourier amplitude terms of the analysis can be converted to power spectral densities, $G\left(\mathrm{gamma}^{2} / \mathrm{Hz}\right)$, by multiplying the square of the spectral amplitude by the constant $4.32 \times 10^{4}$. Details of the spectral analysis representation are given in the Appendix.

The rapid field variations on the days of this study result from geomagnetic storms, the violent and transient disturbance of the Earth's magnetic field lasting from several hours to several days. The special characteristics of the amplitudetime appearance of the geomagnetic storm magnetograms have been reviewed by a number of authors (Akasofu, 1967; Matsushita and Campbell, 1967; RostoKer, 1972; KisABeth and RostoKer, 1974) and will not be repeated here.

In the present study I will emphasize some of the spacial features of the spectral composition of the geomagnetic field for 7 selected days having relatively high levels of geomagnetic activity defined by the daily index $A p$. On the average $50 \%$ of the days in a year have $A p$ values above 7 . About 43 days in a year here $A p>20$ and 10 days usually have $A p>40$. Only about once every other year do $A p$ values reach above 150. Table 1 lists the expected occurrence for my 7 selected days.

The oscillatory period boundaries of this study, at $5 \mathrm{~min}$ and $4 \mathrm{hr}$, were defined by the 2.5-min data sampling period and the large component of the solar-dynamo $(S q)$ and lunar-tidal $(L)$ contributions to the field near $6 \mathrm{hr}$ (MAtsushita and CAMPBell, 1967). This storm spectra study is complement to the earlier work (CAMPBELL, 1976) that emphasized the average daily and seasonal behavior of the geomagnetic field for the same spectral range. The object of the study is to provide a predictable spectral picture of the earth's field changes at the magnetically disturbed times. The present projected uses of such information are to improve the estimation of source fields available for probing the earth's crust, to predict the noise present in certain geomagnetic mapping procedures, to determine the expected induced currents within the Alaskan oil pipeline, and to specify more accurately the global magnetic energy distribution during solar-terrestrial disturbance conditions.

\section{Results}

All the station spectra were scaled at the 5 representative periods $(5,10$, 30,60 , and $120 \mathrm{~min}$ ) indicated in Fig. 1. Spectral slopes were determined for the 5 to $10-\mathrm{min}, 10$ to $60-\mathrm{min}, 60$ to $240-\mathrm{min}$, and 5 to $240-\mathrm{min}$ ranges; vertical lines illustrate the separation of these ranges in Fig. 1. These spectral feature scalings for each active day of Table 1 were identified with the corresponding station geomagnetic locations of Table 2 and plotted in special projection and 
contour formats using available computer techniques. Below I will present first the Fourier component amplitude features of the disturbance days then the spectral slope characteristics.

\subsection{Spectral amplitudes}

Figure 2 illustrates the types of projections and contours available from the analysis as well as the directional field component relationships. The regular 4-hr spectral values at 2-hr steps produce some elongation of the contours along the time axis. The greater density of stations at the higher latitudes contributes to the appearance of variability at the auroral zones. The predominance of high activity at the auroral zone latitudes is clearly discernible even before and after the approximate $8 \mathrm{hr}$ of event maximum activity. Levels of the field at the polar

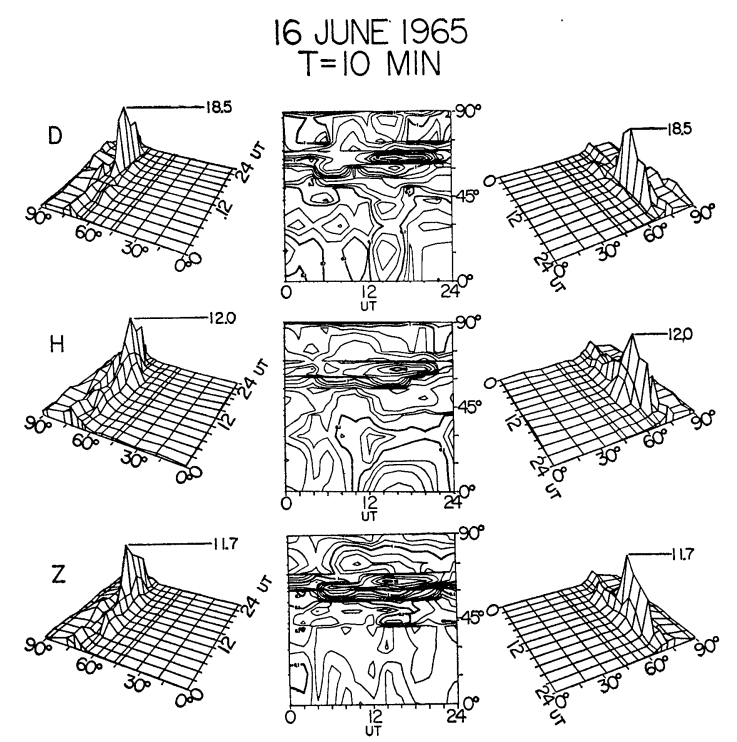

Fig. 2. Latitude-time display of the 10-min amplitude component from the 4-hr spectral analysis of the $D, H$, and $Z$ magnetogram for 16 June 1965. Markers in Universal Time (UT) indicate the central hour of the 4-hr analysis groups which were incremented every $2 \mathrm{hr}$ throughout the event. Geomagnetic latitude markers are in degrees; fixed latitude lines represent the reporting station locations. Peak amplitudes on the two projections (right and left columns) are given in gammas. The maximum scale size of each projection is adjusted to the peak value within the projection. The central coumn in this figure shows the contour plot for the 3 field components. Base level contours at $0.1,1.0$, and 10.0 are given heavy lines. Between these the ligher lines of intermediate contours are at factors of 1.5, 2, 3, 5, and 7 times the lower base contour. 

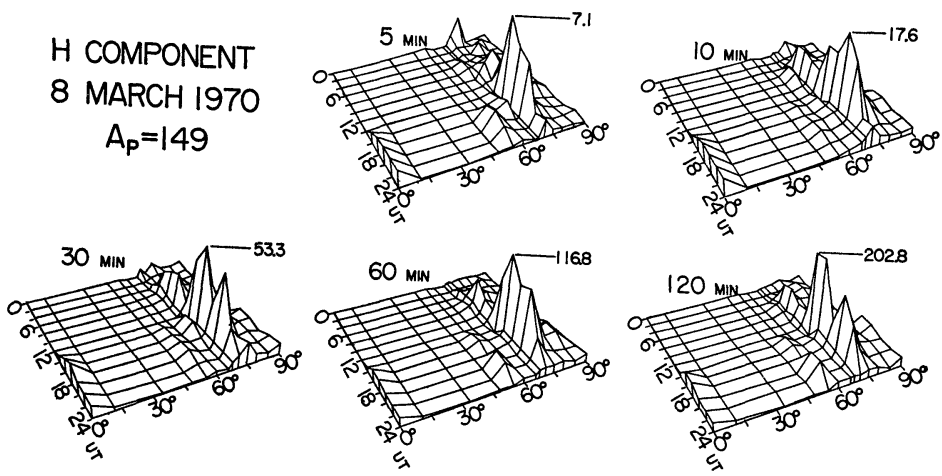

Fig. 3. Spectral amplitudes of 5, 10, 30, 60, and 120-min period Fourier components from the 4-hr analysis sample of the horizontal $(H)$ variation of the geomagnetic field for every $2 \mathrm{hr}$ of Universal Time (UT) covering the disturbances of $8 \mathrm{March}$ 1970, a day of very high daily activity index $(A p=149)$. In these projections the geomagnetic latitude is given in degrees; constant latitude lines indicate the location of the observatories. The maximum amplitude in each period sample is normalized to about the same vertical height for the projections; the amplitude scale can be determined from the peak values (gammas) indicated on each display.

cap are higher than those equatorward of the auroral maxima. The sharp decrease, or "cliff," at the low-latitude side of the auroral zone is more clearly discernible on the contour displays than on the projections. The equatorial enhancement appears mostly on the $H$ component near the Huancayo local noon hours (1700 UT). The $D, H$, and $Z$ components are not too different in general appearance and peak amplitudes. For this event the $D$ component is the largest and the $Z$ component reaches a maximum about $2 \mathrm{hr}$ earlier than the other field components.

Figure 3 illustrates the variation in amplitude at the 5 scaled spectral periods for the $H$-direction field component on 8 March 1970, the most active day of the seven. For each spectral sample the duration of major disturbance is about $10 \mathrm{hr}$. The peak amplitudes increase with period as was apparent in Fig. 1. These peaks are all located at the same latitude although there is some splitting into two separate maxima (more evident on the longer period data) and some variation in the time of the maximum (between 1400 and $1800 \mathrm{UT}$ ). The equatorial enhancement is near the local noon hours at Huancayo.

A summary of the general features of the spectral amplitude analysis of the 7 days is provided in Fig. 4. The average duration of the heightened activity at the auroral latitudes was about $11 \mathrm{hr}$. This did not seem to vary in a noticeable fashion with the $A p$ index, spectral period, or the component direction. The equatorial enhancements favored, but were not restricted to, the daytime hours and were relatively larger for daytime maxima of activity in the auroral 


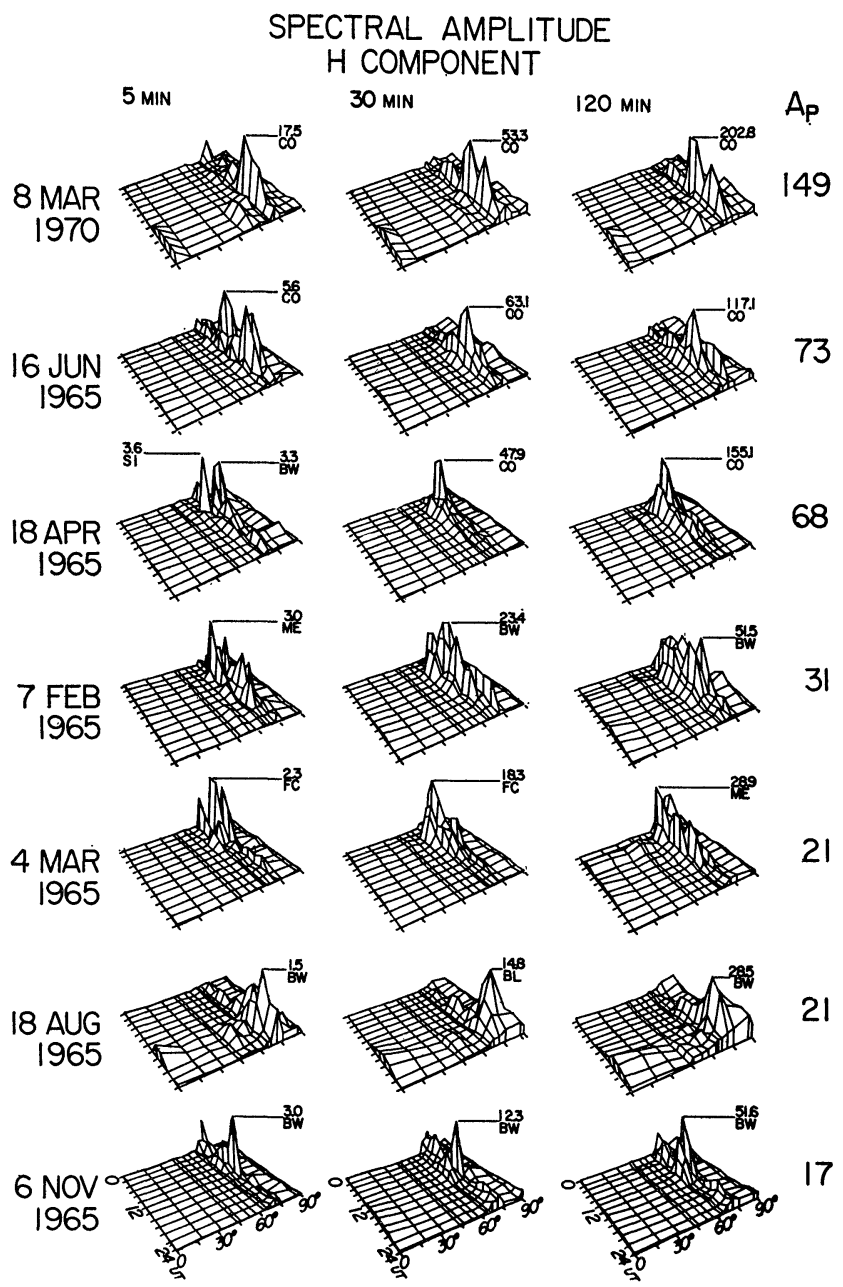

Fig. 4. The 3 columns represent the spectral amplitudes of 5, 30, and 120-min period Fourier components for the 4-hr samples of the horizontal $(H)$ variations in the geomagnetic field every $2 \mathrm{hr}$ of Universal Time (UT in bottom-left scale) for 7 active days (dates at left margin) arranged in order of decreasing daily geomagnetic activity index $A p$ (values at right margin). In these projections the geomagnetic latitudes are given in degrees (bottom-right scale); constant latitude lines indicate the location of contributing observatories. The scale for the maximum amplitude (gammas) of each period sample is normalized to about the same vertical height for all projections. The corresponding station letters (Table 2) for the peak locations are indicated beneath the peak amplitude value. 
zone. The ratio of the equatorial to auroral zone peak amplitudes varied considerably between events without any apparent order. The average time of occurrence of the maximum in activity for a storm was near $1200 \mathrm{UT}$, at the postmidnight to early morning hours for most of the high-latitude station locations. There was a large variation in the time of maximum (peak) for the 7 active periods and no apparent relationship between this time and other measured quantities except for a slight indication that the peak occurred earlier for shorter-period spectral components. The station location for the activity peak seemed to shift to higher latitudes with lower $A p$ index in a fashion similar to that found for the earlier study (CAMPBELL, 1976).

Table 3. Variation of spectral component peak amplitudes, $A_{T}$, with daily $A p$ index. ${ }^{11}$

\begin{tabular}{ccccc}
\hline Period $T(\mathrm{~min})$ & Intercept $a_{0 T}$ & $\mathrm{~d} A_{T} / \mathrm{d} A p a_{1 T}$ & Error $s_{T}$ & Correlation coefficient \\
\hline 5 & 1.65 & 0.0384 & 0.800 & 0.93 \\
10 & 3.19 & 0.111 & 3.09 & 0.88 \\
30 & 14.3 & 0.350 & 13.7 & 0.80 \\
60 & 7.08 & 0.809 & 19.7 & 0.91 \\
120 & 17.6 & 1.35 & 24.9 & 0.94 \\
\hline
\end{tabular}

1) $A_{T}=a_{0 T}+a_{1 T} A p \pm s_{T}$ where $a_{0 T}$ and $a_{1 T}$ are the intercept and slope constants and $s_{T}$ is the standard error of the estimate of $A_{T}$ on $A p$.

The peak values of the spectral component amplitudes occur at the auroral latitudes. Table 3 gives the linear fit to the data points representing the spectral component peak amplitudes, $A_{T}$, and corresponding daily activity indices, $A p$, for the 7 days at 5 different spectral periods, $T$. The correlation coefficients obtained are all quite high. The minimum correlation occurs for the 30 -min spectral component; maximum correlation for the 5 and 120-min components. The rate of change of amplitudes with $A p$ increases with increasing $T$. The values of $a_{0 T}$ listed in the table indicate that at low $A p$ the 30-min spectral component amplitudes may be larger and the 60-min spectral amplitudes may be smaller than expected for a usual average spectra with amplitudes increasing smoothly with period. However, in the region below about $A p=15$ the spectra probably move away from substorm patterns into a low-activity domain; at $A p$ values of about 5 and below the special quiet level spectra occur. The equations of Table 3 were obtained for the $A p$ range from 17 to 149; although I would not recommend extrapolation to values below $A p=15$, I expect that the substorm spectral behavior would extend to at least $A p=250$.

The peak power spectral density, $G(T)$, at any period, $T$, may be obtained from

$$
G(T)=4.32 \times 10^{4}\left(a_{0 T}+a_{1 T} A p \pm s_{T}\right)^{2}
$$


where the constants $a_{0 T}, a_{1 T}$, and $s_{T}$ are given in Table 3. From a distribution of 11 years of geomagnetic indicies by Doeker (unpublished data, 1974), I found that the number of days per year, $N$, on which the daily $A p$ values exceeded any given index level can be approximated by the relationship

$$
N=4.14 \times 10^{4} A p^{-2.25}
$$

for a range of $A p$ values from about 15 to 150 . Equations 1 and 2 and Table 3 allow one to establish how many days per year the peak power spectral density at a particular Fourier component period will exceed any specified amplitude at the auroral zone latitudes.

For a particular active day the collection of the daily average spectral component amplitudes at each station (Fig. 5) provides another view of the average latitude distribution of the field. The daily average values of the spectral amplitude at the station where the peak activity occurs is 0.32 of the 4-hr sample peak amplitude value (standard deviation $=0.08$ ). The ratios of the daily average

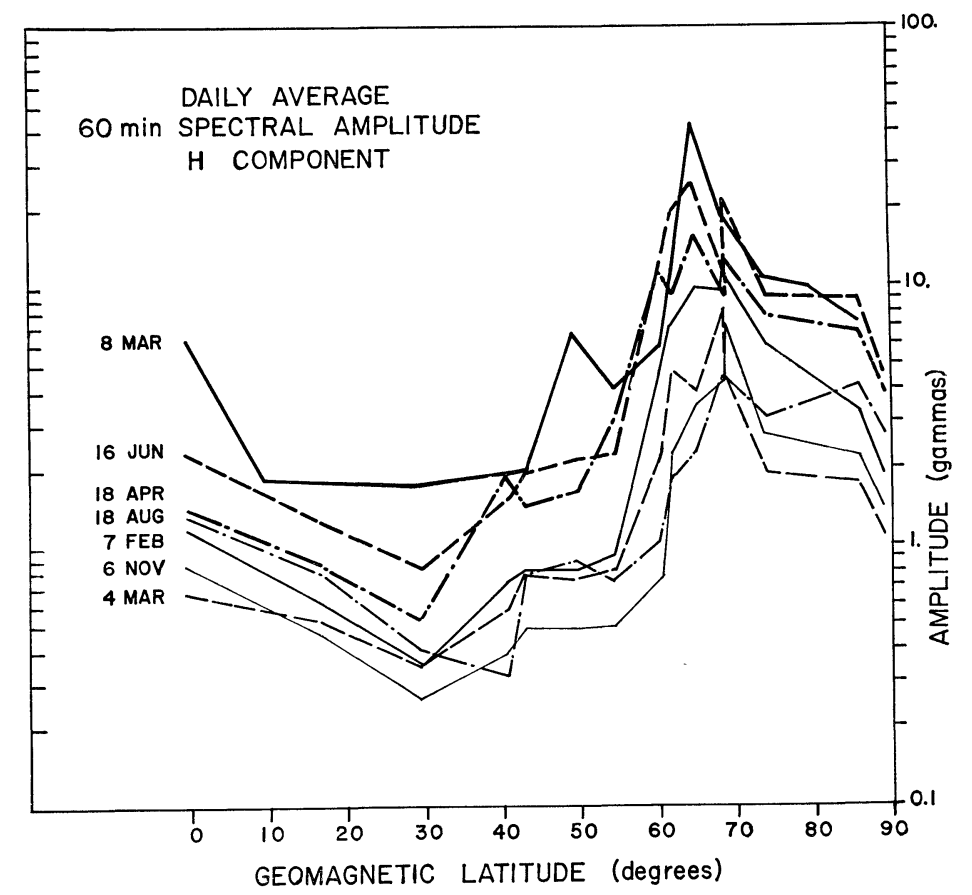

Fig. 5. Latitude variation of the daily average of the 60-min Fourier component spectral amplitudes (gamma) for the $H$-component field direction. At each latitude of station location the average of the spectral amplitudes was determined separately for the 7 events given by the dates in the figure. Heavier lines indicate higher $A p$ indices (Table 1). 
of auroral zone maximum to low-latitude minimum values for the 60 -min amplitudes of the disturbances in Fig. 5 average 25 and showed no apparent relationship to $A p$. For the various spectral period samples this ratio varied as $T^{-0.42}$. That is, the spectral amplitudes at the longer periods do not show as great a latitudinal variation as the amplitudes at the shorter periods.

\section{5-240 MIN SPECTRAL SLOPE 16 JUNE 1965}
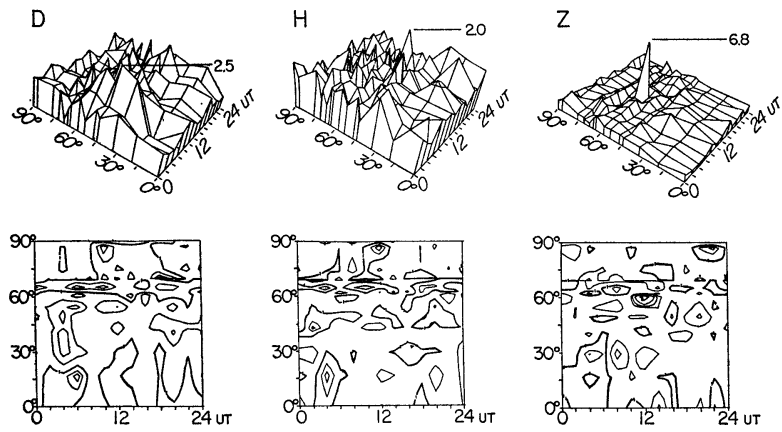

Fig. 6. Spectral slope for amplitudes of the 5 to 240 -min period range in the event of 16 June 1965. Three columns represent $D, H$, and $Z$ field components. Top row is the projection views with the vertical scale size of each display determined by the maximum slope value (indicated). Geomagnetic latitude (degrees) and time (UT hour) are shown. Bottom row is the corresponding contour displays. Heavy lines are base level contours at 0.1 and 1.0 slope values. Lighter lines are contours at 1.5, 2, 3, 5, and 7 times the lower base level contour. Note the anomalous peak value on the $Z$-projection.

\section{H SPECTRAL SLOPE $8 \mathrm{MARCH} 1970$}
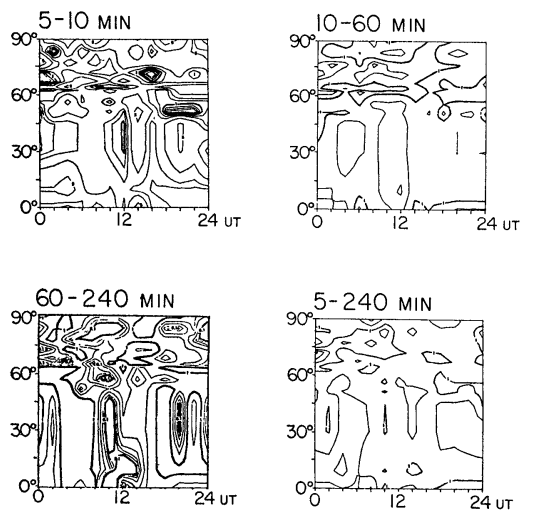

Fig. 7. Spectral slope for amplitudes of 5 to 10,10 to 60,60 to 240 , and 5 to $240-\mathrm{min}$ period ranges for the $H$ component of field in the event of 8 March 1970. These contour displays are in geomagnetic latitude (degrees) and time (UT hour) coordinates. Heavy lines are base level contours at 0.1 and 1.0 slope values; the lighter contours are at 1.5, 2, 3, 4, and 7 times the lower base level contour. 


\subsection{Spectral slope}

An analysis of the spectral slopes can indicate whether there is a systematic variation of the distribution of the relative amplitudes in a spectral display with changing latitude. In the earlier discussion of Fig. 1 the selected period ranges for the analysis of this slope were indicated. Figure 6 is a view of the 5 to 240-min period range results for the three orthogonal field component directions. Because each projection display is scaled to an equivalent maximum value, the anomalously large $Z$-component slope of 6.8 at Sitka (1200 UT) suppresses the appearance of the scale size for all other measurements on that date and thereby emphasizes the typically small variations in the slope at most latitudes during the event. The contour displays in the figure show that the spectral slope differences between geomagnetic field components are not great.

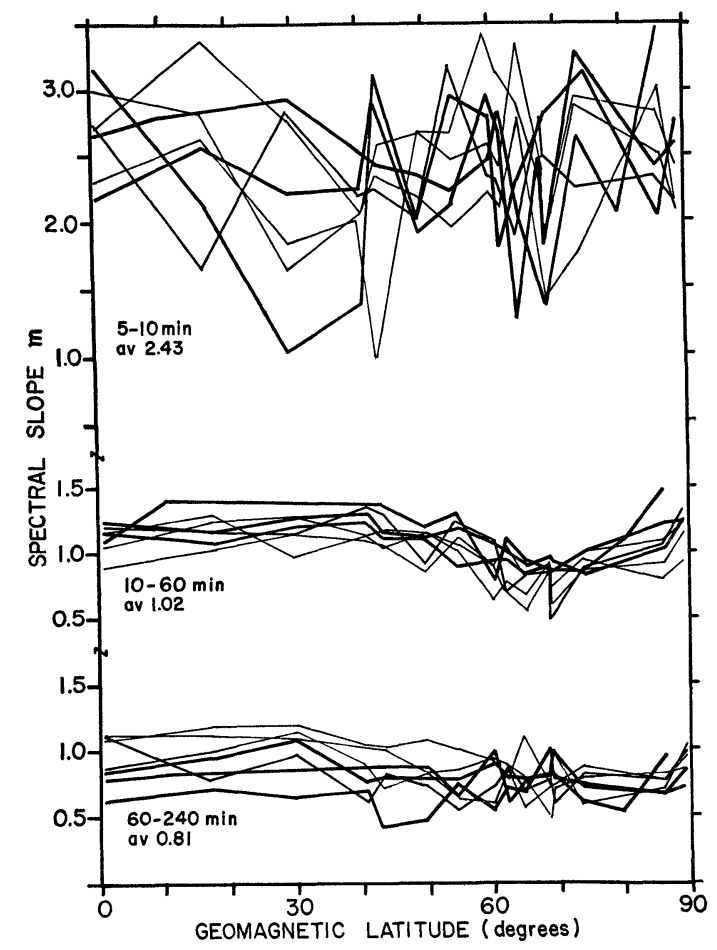

Fig. 8. Spectral slope, $m$, for amplitudes of the 3 period ranges ( 5 to 10 , 10 to 60 , and 60 to $240 \mathrm{~min}$ ) for the $H$ component of field for all 7 days (Table 1) with $m$ values averaged throughout the day of each event at each geomagnetic latitude of the stations given in Table 2. The average values (av) of all measured slopes in each day are indicated. The 3 most active and 3 most quiet $A p$ days (Table 1) are indicated by the heaviest and lightest lines respectively. 
That the slope, $m$, varies for different sections of the spectra can be observed in the example of Fig. 7. The highest values, with $m$ between 1 and 10 (average 2.4 ), are observed in the 5 to 10 -min period range whereas the lowest values, with $m$ between 0.1 and 1.0 (average 0.8 ), are observed in the 60 to 240-min period range. The midrange and full-spectra slopes showed values generally near 1.0.

The variations in the 10 to 60 -min slope values were usually out of phase with the longer-period range slope variations. A possible way for this phenomenon to occur would be for the spectral amplitude variations near 60-min period to be most responsible for defining the two adjacent slopes. In such an arrangement the increases of activity would couple the increase in 10 to 60 -min slope with the decrease of 60 to 240 -min slope.

The daily average values for constant latitude sections through the slope contour displays are given in Fig. 8. The great variability in the slopes at the shorter-period range may be due to aliasing effects which can be expected below $T=6.25$ min (OTNES and ENochson, 1972) or due to the unreliability of data at low amplitudes near the resolution level of the scalings. This figure also shows that there is a decrease of spectral slope at the auroral zone for the 10 to 60-min range for all disturbed days; the average value for stations between $60^{\circ}$ and $70^{\circ}$ in the figure is 0.86 . Such a decrease is the opposite of what one would at first expect because the slope of this range is known to increase with increasing activity and the larger amplitudes do occur at the auroral zone. The

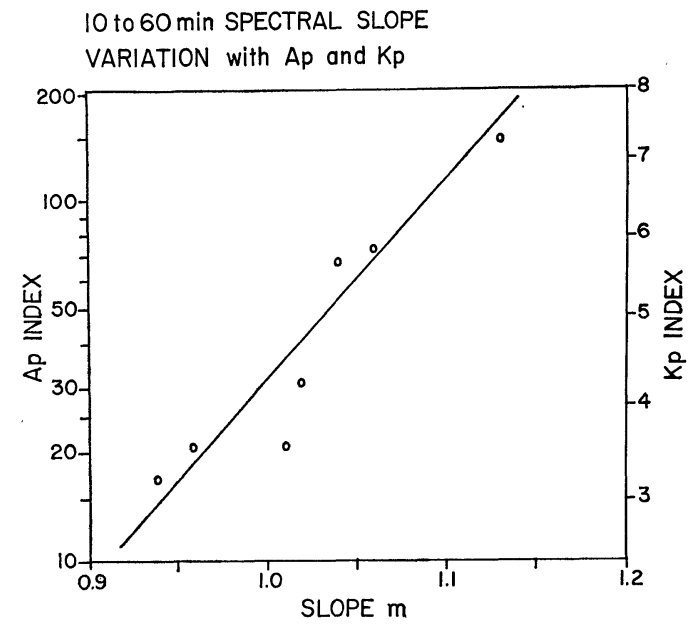

Fig. 9. Variation, with $A p$ and $K p$ geomagnetic indices, of the daily average of 10 to 60-min spectral slope of all stations (Table 2) for an event with the corresponding daily $A p$ (Table 1 ) or $K p$ indices. 
observed decrease means that the ratio of shorter-period amplitudes to longerperiod amplitudes is larger at the auroral zone than at other latitudes for all levels of activity in the 10 to 60 -min period range. The spectra of this region may be more representative of the field aligned currents from the magnetosphere that dominate the auroral zone.

Figure 9 shows the variation of the daily average 10 to 60 -min spectral slopes with the planetary geomagnetic index $K p$ by indicating the relationship of this index to $\log A p$. The best fitting straight line (correlation coefficient= 0.94 ) is given in the figure by

$$
m_{10,60}=0.75+0.169 \log A p \pm 0.02 .
$$

Thus, as the geomagnetic activity level increases the longer-period field fluctuations increase in amplitude more rapidly than the shorter-period fluctuations for this range. The 5 to 10 -min period spectral range showed a much poorer correlation with $A p$ (correlation coefficient $=0.15$ ) and the 60 to $240-\min$ period range slope decreased with increasing $A p$ (correlation coefficient 0.52).

\section{Discussion}

Indices such as $A p$ are obtained from the extremes of magnetic field amplitude variations over a restricted time period at a special distribution of geomagnetic observatories. Such indicies provide a measure of the degree of intensity of the geomagnetic disturbances at the Earth's surface. In the present paper these indices were used to select a variety of active days for determining the uniform and predictable changes in the spectral characteristics of geomagnetic variations with time and location. Therefore, it was expected that the spectral amplitudes would increase with $A p$, the questions were how much, at what rate, at what frequency, and where was the variation. The established statistics of occurrence for $A p$ index levels provided a broad contex into which the 7 selected days were placed in terms of the probable number of such events that may be expected in an average year (Table 1).

The field amplitudes generally increase with period and the latitudinal display of the amplitudes showed such features as an auroral zone maximum, midlatitude minimum, and equatorial enhancement. These are all well-known characteristics that need not be reviewed here. The spectral component amplitudes and slopes to be expected for the various substorm levels was the focus of the present paper and the results were expressed in the figures, tables, and formulas.

One surprise in the analysis was the depression of the 10 to 60 -min spectral slope between about $55^{\circ}$ and $75^{\circ}$ latitude. This anomaly, being associated with 
the auroral zone and independent of activity level (Fig. 8), means that the shorter-period variations contribute proportionally more to the surface magnetic spectra in those regions closer to the source where the global amplitudes are higher than in other regions. It seems that some shorter-period field variations either arise at too high an altitude to couple into the ionospheric current system or are selectively attenuated a short distance from the source latitude.

Spectra of the geomagnetic field variations have been published by a number of observers for parts of the spectral range of this study (DAvidson, 1964; Dubrovskiy and Kamarenko, 1971; Best et al., 1972; Campbell, 1973; Morgan and Lanzerotti, 1973; Lanzerotti and Surkan, 1974; Campbell, 1976). In the present study, however, the special feature was the view of the latitudinal variation of geomagnetic spectra for differing activity levels.

There are some shortcomings in the analysis. Three of which I would like to mention here. The first is the problem of longitude. Although all stations were located in the American hemisphere, those at larger differences in longitude near or in the auroral zone will show the substorm maximum occurring at different hours. Near the pole, local time differences lose their meaning and at low latitudes the diurnal changes in ionospheric conditions are gradual enough that up to several hours time difference between stations has little, if any, noticeable effect upon the spectral amplitudes in this study. For an extreme example of the longitude difficulty I purposely included both Barrow and Fort Churchill in the station sample. These two stations are at about the same geomagnetic latitude but differ in local time by about $4 \mathrm{hr}$ (Table 2). The difference in spectral amplitude for these stations appears as a noticeable irregularity between the $68^{\circ}$ and $69^{\circ}$ latitudes in the figures but does not affect the major conclusions of this paper.

The second shortcoming concerns the spectral sample size. To resolve the longer period changes a data block of $4 \mathrm{hr}$ was chosen and this sample was advanced at 2 -hr intervals through the event. Such a process necessarily provides some smoothing of amplitude variations. From time to time singular frequency peaks at a number of stations did appear simultaneously in the spectral amplitude displays; however no consistent resonant frequencies were found on the disturbed days. How the long sample length may have contributed to this negative result is not clear.

The third shortcoming was the small number and type of active days. More events over a variety of seasons and parts of the solar cycle would have greatly improved the study.

I thank Bernice Bender of NOAA for her expert assistance with the computer programming in this study and Carol Stewart of USGS for her dedicated help with the analysis. I also thank the staff of World Data Center A for their special scaling of station magnetograms and for 
supplying all the original digital tape records; to Carl Abston, Joe Allen, and Leslie Morris I am especially grateful.

\section{APPENDIX}

Geomagnetic field variations have significant amplitude contributions through a large and continuous range of frequencies. Such spectra meet the conditions for fluctuating noise analysis (ScHWARTz, 1959). A Fourier expansion of the amplitude-time variations of the field gives

$$
f(t)=\frac{c_{o}}{2}+\sum_{n=1}^{\infty} c_{n} \cos \left(\omega_{n} t+\theta_{n}\right),
$$

where $f(t)$ is the geomagnetic field component amplitude in gammas varying with time $t, c_{o} / 2$ is the offset level, and $c_{n}$ is the $n$th Fourier component term corresponding to frequency $\omega_{n}(\operatorname{radian} / \mathrm{sec})$ and phase $\theta_{n}(\operatorname{radian})$. The power spectral density $G\left(\omega_{n}\right)$ in gamma $^{2} / \mathrm{Hz}$ is then defined as

$$
G\left(\omega_{n}\right)=\frac{\tau}{2}\left|c_{n}\right|^{2}
$$

where $\tau$ is the analysis interval in seconds and is given by

$$
\tau=N_{\mathrm{p}} \Delta t
$$

where $N_{\mathrm{p}}$ is the number of scaled values in the sample and $\Delta t$ is that time between samples. Thus we may write

$$
G(\omega)=\frac{N_{\mathrm{p}} \Delta t}{2}\left|c_{n}\right|^{2} .
$$

The spectral amplitudes discussed herein are the values of $\left|c_{n}\right|$ in gammas. These amplitudes may be converted to the power spectral density $G\left(\omega_{n}\right)$ by squaring the $\left|c_{n}\right|$ and multiplying by the factor $4.32 \times 10^{4}$ (CAMPBELL, 1976).

\section{REFERENCES}

Akasofu, S.I., Polar and Magnetospheric Substorms, Springer-Verlag, New York, 1967.

Best, A., L.L. Vanyan, V.G. Dubrovskiy, S.A. Kramarenko, and G.R. Lemann, Latitudinal distribution of geomagnetic disturbances, Geomag. Aeron., 12, 432-434, 1972.

CAMPBelL, W.H., Spectral composition of geomagnetic field variations in the period range of $5 \mathrm{~min}$ to $2 \mathrm{hrs}$ as observed at the Earth's surface, Radio Sci., 8, 929-932, 1973.

CAmpbell, W.H., An analysis of geomagnetic variations having periods from $5 \mathrm{~min}$ to $4 \mathrm{hrs}$, J. Geophys. Res., 81, 1369-1390, 1976.

Cooley, J.W., P.A.W. Lewis, and P.D. Welch, The Fast Fourier Transform and its applications, IEEE Trans. Educ., E-12, 27-34, March 1969.

Davidson, M.J., Average diurnal characteristics of geomagnetic power spectrums in the period range 4.5 to 1,000 seconds, J. Geophys. Res., 69, 5116-5119, 1964. 
Dubrovskiy, V.G. and S.A. Kamarenko, Spectral characteristics of the terrestrial and interplanetary magnetic fields in the frequency range 1 to $10^{-4} \mathrm{~Hz}$, Geomag. Aeron., 11, 884-889, 1971.

Kisabeth, J.L. and G. Rostoker, The expansive phase of magnetospheric substorms. 1. Development of the auroral electrojets and auroral arc configuration during a substorm, J. Geophys. Res., 79, 972-984, 1974.

Lanzerotti, L.J. and A.J. Surkan, ULF power near $L=4$ : Relationship to Fredericksburg K index, J. Geophys. Res., 79, 2413-2419, 1974.

Matsushita, S. and W.H. Campbell, Physics of Geomagnetic Phenomena, Academic Press, New York, 1967.

Morgan, C.G. and L.J. Lanzerotti, Enhancements in geomagnetic power spectra in the frequency band 1.6 to $6.8 \mathrm{MHz}, J$. Geomag. Geoelectr., 25, 27-38, 1973.

Otnes, R.K. and L. Enochson, Digital Time Series Analysis, pp. 1-40, John Wiley and Sons, New York, 1972.

Rostoker, G., Polar magnetic substorms, Rev. Geophys. Space Phys., 10, 157-211, 1972.

Schwartz, M., Information Transmission, Modulation and Noise, pp. 266-282, McGraw-Hill, New York, 1959. 